\title{
An Hierarchical Approach Towards Road Image Segmentation
}

\author{
Ashfaqur Rahman \\ Intelligent Sensing and Systems Lab CSIRO \\ Hobart, Australia \\ Email: ashfaqur.rahman@csiro.au
}

\author{
Brijesh Verma \\ Central Queensland University \\ Rockhampton, Australia \\ Email: b.verma@cqu.edu.au
}

\author{
David Stockwell \\ Transport and Main Roads \\ Queensland, Australia \\ Email: David.R.Stockwell@tmr.qld.gov.au
}

\begin{abstract}
The segmentation of road images from vehicle mounted video is a challenging and difficult problem. One of the problems is the presence of different types of objects and not all objects are present in the same frame. For example, road sign is not visible in all frames. In this paper, we propose a novel framework for segmenting road images in a hierarchical manner that can separate the following objects: sky, road, road signs, and vegetation from the video data. Each frame in the video is analysed separately. The hierarchical approach does not assume the presence of a certain number of objects in a single frame. We have also developed a segmentation framework based on SVM learning. The proposed framework has been tested on the Transport and Main Roads Queensland's video data. The experimental results indicate that the proposed framework can detect different objects with an accuracy of $95.65 \%$.
\end{abstract}

Keywords- road image segmentation, video indexing, SVM.

\section{INTRODUCTION}

An integrated part of an intelligent transportation system is a computer vision based system that analyses the images captured by cameras mounted on top of the vehicle. It is required that an automated vehicle is capable of detecting obstacles, road bumps, falling trees and at the end a safe road to travel along. Image segmentation solutions are a suitable match for this problem. The transport images to be segmented are however different to conventional images used in contemporary segmentation problems: (i) most of the on-road objects like trees, vegetation, etc. do not have any specific shape, (ii) the objects are scattered and mingled with each other (e.g. sky, poles, and trees), (iii) A single object can have different colour distribution in different images (e.g. vegetation can be dry white, yellow, green and brown in colour). Altogether it poses a significant challenge to segment road images using conventional image segmentation algorithms.

A number of researchers have proposed algorithms to separate objects in road images. A road segmentation and road type identification approach was proposed in [1] for Autonomous Land Vehicle (ALV) navigation system based on a new histogram calculation method that makes a statistic of the pixels under some special direction. The authors in [2] presented an algorithm to separate road and non-road in nonstructured road images at the presence of shadows. A morphological approach towards separating road and non-road objects was presented in [3] based on an optimizing threshold segmentation algorithm. A method was presented in [4] for identifying and separating road signs in high resolution images.
A comprehensive study on the use of colour spaces to separate road signs was presented in [5]. An algorithm for separating road from non-road objects and on-road obstacles was presented in [6] using fast watershed transformation. An image segmentation method for the recognition of texture-based objects in a road environment scene was presented in [7]. A colour image segmentation approach based on an improved Restricted Coulomb Energy neural network was presented in [8] for road/traffic colour image segmentation. A road extraction method using the one-class support vector machine (SVM) was presented in [9].

The algorithms mentioned above are suitable for high quality images in general. Moreover the number of objects to be separated is confined to either (i) road and non-road objects or (ii) road signs only. In this paper, we present a research work to segment a road image into a number of objects including (i) road, (ii) sky, (iii) green signs, (iv) light blue signs, (iii) speed signs, (iv) yellow signs, and (v) vegetation. We have used road images captured on Australian countryside highways and the images were provided by Transport and Main Roads (TMR). The novelty of the work lies in the fact that the proposed hierarchical segmentation algorithm is capable of separating a large set of objects on road images. The quality of captured images being low also imposes a challenge to the segmentation algorithm. We have also investigated another learning based framework to segment road images.

The paper is organized as follows. The step-by-step segmentation algorithms for separating the abovementioned objects are presented in Section II. The images and computing platforms used in the experiments are detailed in Section III. Findings and related discussions are presented in Section IV. Finally Section V concludes the paper.

\section{PROPOSED SEGMENTATION FRAMEWORK}

Given a road image the proposed segmentation algorithms works in a hierarchy as presented in Figure 1. The learning based framework (Figure 1(a)) first segments images using $k$ means clustering algorithm. Noise filtering is applied next to eliminate noisy blobs. Features are then extracted from each segment. The above steps are common for both training and testing. During training classifiers are trained on the feature set of each segment. During testing feature sets are classified into the following categories by the classifiers: lane, vegetation, sky, road surface, white board, yellow board, board blue, board green, red sign, gray pole, black board, white pole. The next 
task is to combine multiple segments into target objects like road sign, road, etc.

With the hierarchical approach (Figure 1(b)) a few pre processing steps are added at the beginning to assist the following segmentation algorithms. The road images are extracted first. From the remaining images sky portion is separated. The different road signs are extracted next and remaining of the image is declared as vegetation. We call the proposed framework hierarchical due to its inherent nature. All these steps are detailed next.

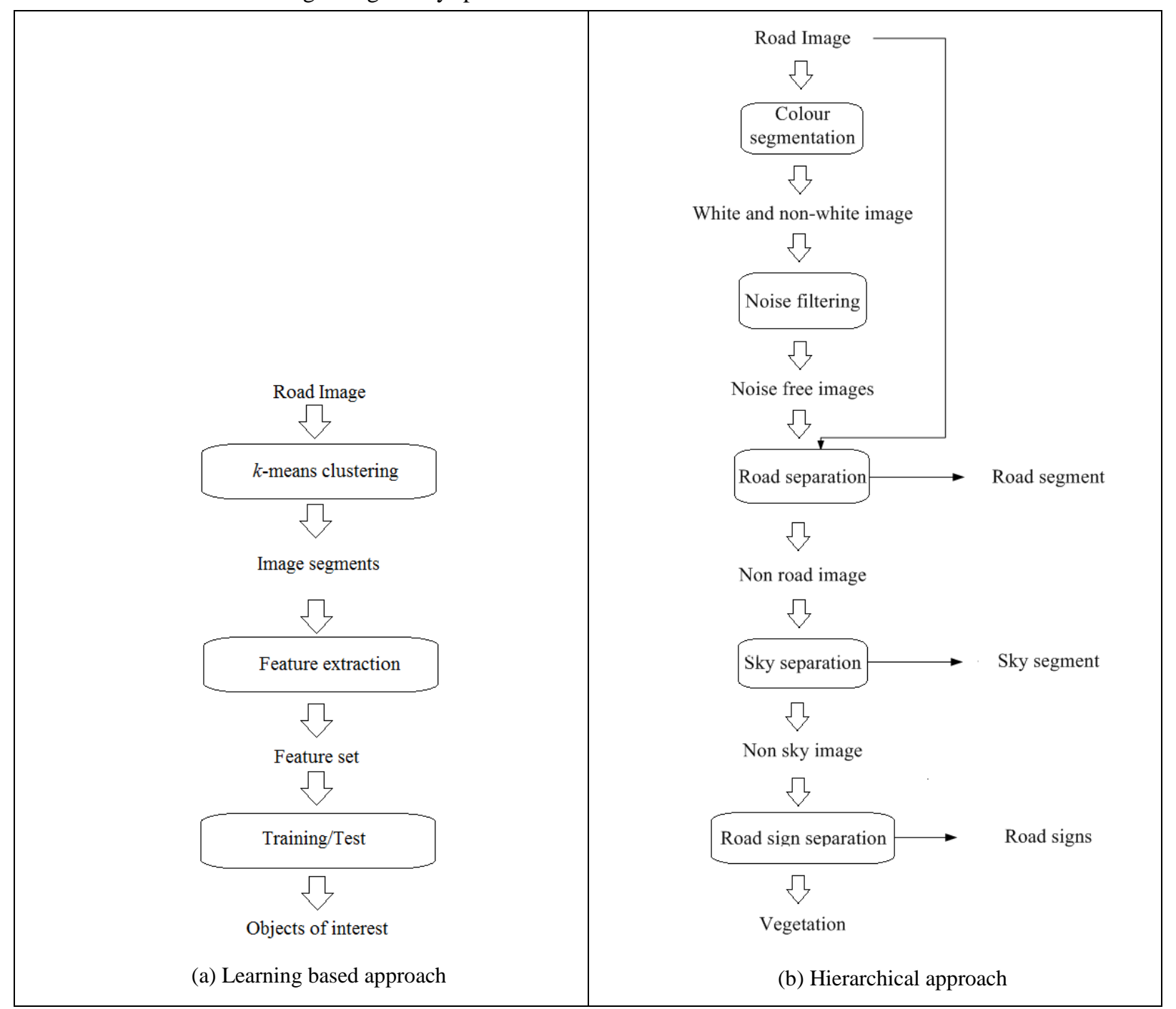

Figure 1: Road image segmentation algorithm

\section{A. Colour Segmentation}

Given a road image we first segment it into two colour channels: white and non-white. We have used $k$-means clustering algorithm for this purpose with $k=2$. This segmentation process (Figure 2) provides some structural information about the road scene. The white segment contains lanes, dry vegetation, white board of road signs and sky. The non-white segment contains road, coloured portion of road signs (e.g. green, blue, and red) and green vegetation. This masks (i.e. non-black portion in an image) corresponding to these two segments are used in later stages to identify the objects mentioned earlier.

\section{B. Noise Filtering}

The noise filtering (Figure 3) is applied to the masks corresponding to both white and non-white images to get rid of small scattered coloured portions in images. This reduces the possible contenders for the target objects.

\section{Road Extraction}

Given the mask corresponding to the white segment the road extraction process starts by finding the white lanes from bottom left and bottom right corner of the image. The search then continues vertically until it finds another white region (i.e. the dry vegetation). The search process then follows the edge of dry vegetation to identify the boundary of the road. Anything to the left and right of the road area are removed 
from the mask. A noise filtering is applied at the end to get rid of disconnected non-road blobs. The steps are shown in Figure 4. The road is subtracted from the original image and the successive steps work on the remaining image.

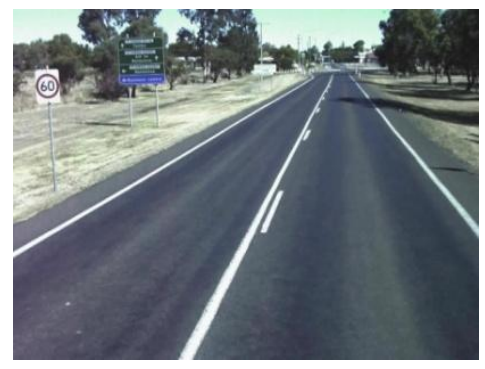

(a) Original image

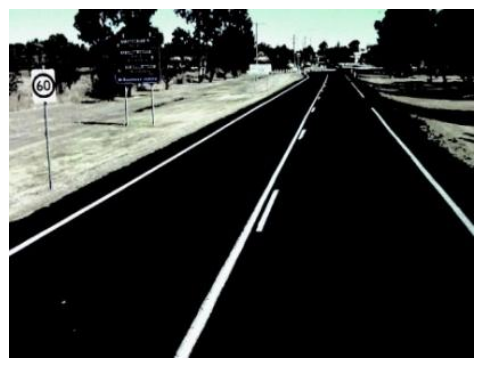

(b) white segment

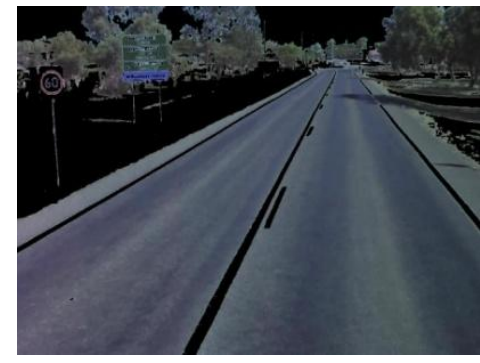

(c) non-white segment

Figure 2: Image segmentation into two colour channels: white and non-white.

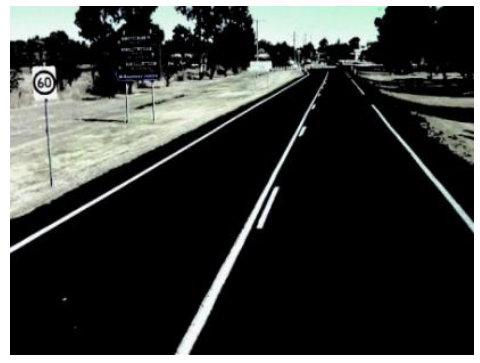

(a) white segment

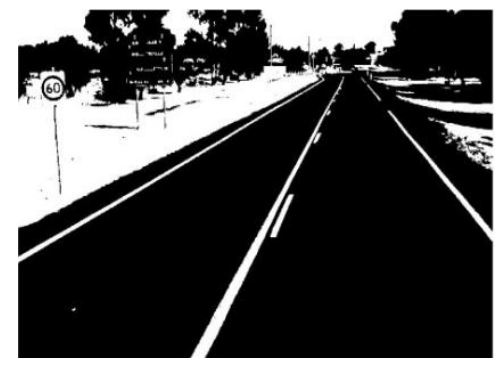

(b) corresponding mask

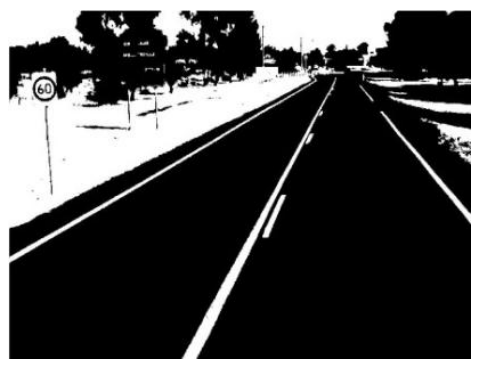

(c) noise filtered mask

Figure 3: Noise filtering to reduce insignificant blobs.

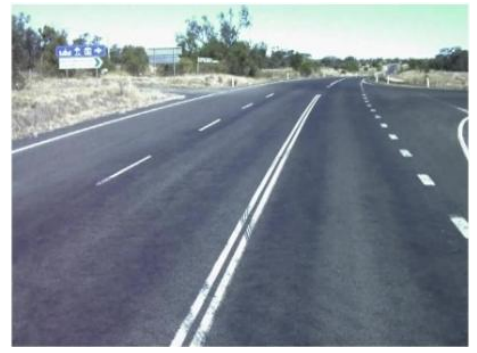

(a) Original image

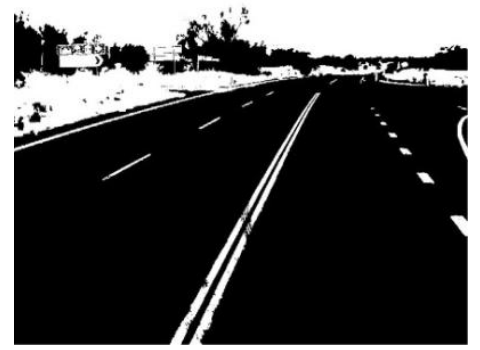

(b) noise filtered white segment

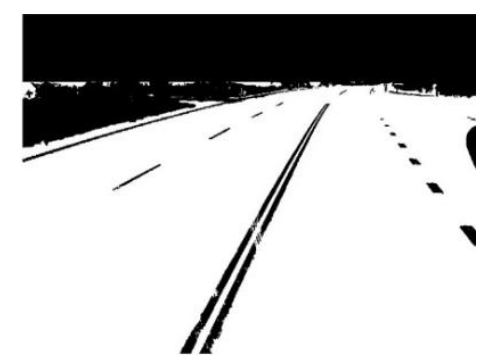

(c) trimming non-road blobs

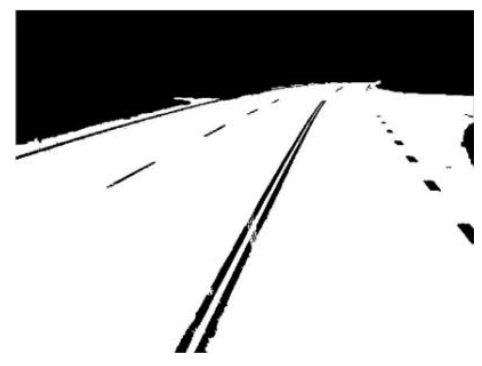

(d) noise filtering

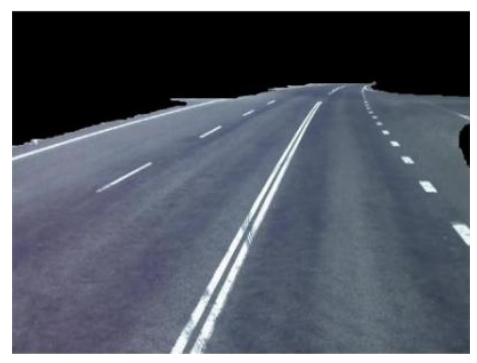

(e) road segment

Figure 4: Road segment extraction. 


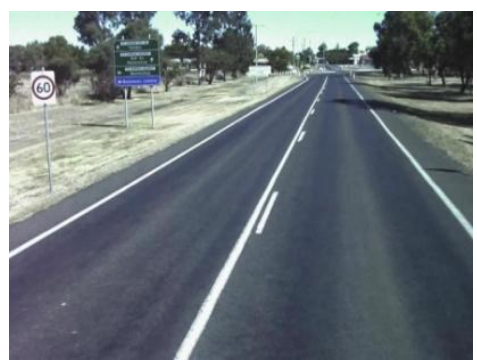

(a) Original image

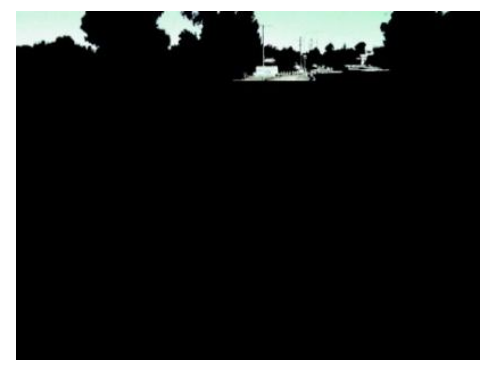

(c) Sky regions connected to the top edge

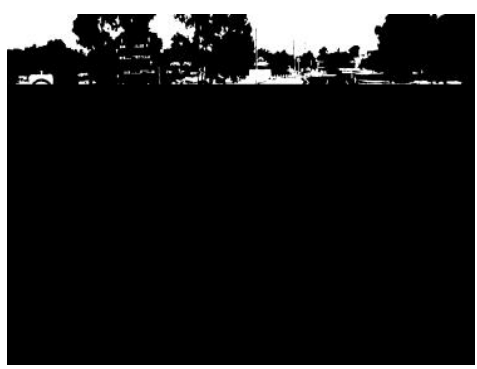

(c) White segment at the top quarter

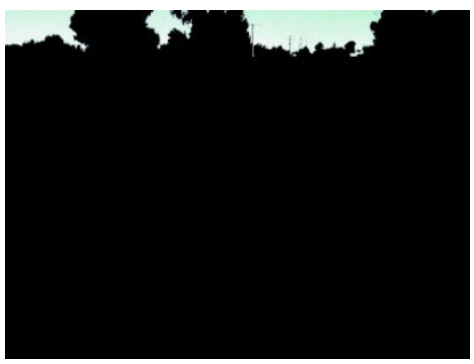

(d) Noise filtered final sky segment

Figure 5: Sky segment extraction.

\section{Sky Extraction}

To separate the sky region we confine the search to the top quarter of the image. The sky is extracted from the white segment by finding blobs that are connected to the top edge of the image. The process however ends with some noises like poles that are connected to the sky. We then divide the region into a rectangular grid of equal size blocks $(64 \times 64)$. Colour and texture histograms are extracted from each block. The blocks are labelled as sky, non-sky, and background. An SVM classifier is trained on the features extracted from the blocks for classification purposes. The test images show an accuracy of $97 \%$ for detecting the sky blocks. The steps are explained in Figure 5.

\section{E. Road Sign Extraction}

Road signs are extracted from the remaining image after subtracting road and sky region from the original image. We extracted four types of road signs from the image: green signs, light blue signs, yellow signs, and speed signs. We used colour ranges to extract regions from the images. We then found the boundaries of the regions. The regions are then filtered by shape information. The green and light blue signs are filtered using rectangular shapes. The yellow signs are filtered using rhombus shapes. The speed signs are composed of red circles surrounded by white regions. After extracting the red circles surrounding white boundaries are sought for in the white segment of the image. The colour ranges used in the experiment are shown in Table 1. After removing the road, sky and road signs the remaining image is declared as vegetation.

\section{EXPERIMENTAL PLATFORM}

The images used in the experiment were captured from the video provided by TMR. The original videos were in avi format with MJPEG codec. For the sake of using them in MATLAB we converted the videos into avi format with MPEG codec using Prism [10]. The extracted frames were saved as JPEG files for later processing. The images have a resolution of $960 \times 1280$. We have used MATLAB for processing the images. For classifying blocks in the sky segment we have used LibSVM [11] implementation of Support Vector Machine (SVM). We have also used SVM in the learning based segmentation framework.

\section{RESULTS AND DiSCUSSION}

We have conducted experiments on a real-world image database from TMR Queensland to evaluate the effectiveness of the proposed approach.

The segment classification matrix on training and test sets using the learning based segmentation framework is presented in Figure 6. The training set accuracy was $96.63 \%$ and the test set accuracy was $86.73 \%$. There has been some misclassification among the objects as can be seen in Figure 6. A major reason for such misclassification is class imbalance. After segmentation some classes like poles, red signs, and boards has a small number of instances compared to other classes.

Table 1: Colour ranges used in segmentation

\begin{tabular}{lc}
\hline Colour & Range \\
\hline Dark Green & $\mathrm{H}>0.3$ and $\mathrm{H}<0.5, \mathrm{~S}>0.2$ and $\mathrm{S}<0.5$, \\
& $\mathrm{V}>0.2$ and $\mathrm{V}<0.7$ \\
Light Blue & $\mathrm{H}>=0.50$ and $\mathrm{H}<=0.70, \mathrm{~S}>0.40$ and \\
& $\mathrm{S}<0.70, \mathrm{~V}>0.50$ \\
Yellow & $\mathrm{H}>0.1$ and $\mathrm{H}<0.20, \mathrm{~S}>0.18, \mathrm{~V}>0.9$ \\
Red & $\mathrm{H}>=0.40, \mathrm{~S}<0.3$ \\
\hline
\end{tabular}


The experiments using the proposed hierarchical segmentation framework under different scenarios are presented in Figures 711. The road, sky and speed sign were successfully extracted from the image in Figure 7. Note that the vegetation contains some white boards. At this stage the framework does not include any step to eliminate white boards. Also note that some part of the road segment is visible in the vegetation image. This is due to the fact that we put an upper limit on the height of the image up to which the road segments are expected.

The road, sky and light blue signs were correctly segmented from the image in Figure 8. Some part of the road segment is also visible in the vegetation image. However this is due to the fact that the left lane merges with the vegetation and the search process for the road segment stops there. Most of the segments are also correctly identified from the image in Figure 9. The sky segment however contains some part of the pole. The colour and texture distribution features show high similarity between parts of poles and sky. Due to this the pole segment is misclassified as the sky segment. Also note that a yellow sign was not detected. This is due to the fact that the size of the yellow blob was small and the noise filtering step eliminates it from the search process.

Some part of the road surface in Figure 10 is visible in the vegetation segment. This is due to the upper limit discussed earlier. The yellow signs were correctly detected. Note that vegetation segment also contains some vehicles. The present framework does not provide any mechanism to detect on-road vehicles. The image in Figure 11 contains a number of different speed signs and all of them were successfully detected. We tested our framework on images containing a total of 23 different objects. The proposed framework was able to detect 22 objects correctly leading to a $95.65 \%$ accuracy.

\section{CONCLUSION}

In this paper, we have presented a novel hierarchical framework for segmenting road images into: sky, road, speed sign, blue sign, green sign, yellow sign, and vegetation from the video data. The proposed framework has been tested on the road images provided by TMR. The experimental results show that the proposed framework can detect different road objects with an accuracy of $95.65 \%$. The hierarchical framework does not take into consideration the white boards and vehicles. In future we aim to segment the images further into white boards and on road vehicles.

\section{REFERENCES}

[1] L. Zhang, E. -Y. Wu, "A Road Segmentation and Road Type Identification Approach Based on New-Type Histogram Calculation," $2^{\text {nd }}$ International Congress on Image and Signal Processing, (CISP), pp.1-5, Oct. 2009.

[2] C. F. Fan, Z. M. Li, X. Q. Ye, and W. K. Gu., "An Adaptive Method of Color Road Segmentation," In Proceedings of the 7th International Conference on Computer Analysis of Images and Patterns (CAIP), Springer-Verlag, London, UK, pp. 661-668, 1997.

[3] G.-B. Xu, S.-Y Xie, and Y. -X Yin, "An Optimalizing Threshold Segmentation Algorithm for Road Images Based on Mathematical Morphology," Third International Symposium on Intelligent Information Technology Application (IITA), vol.2, pp. 518-521, 2009.

[4] H. Fleyeh, "Color detection and segmentation for road and traffic signs," IEEE Conference on Cybernetics and Intelligent Systems, vol.2, pp. 809-814, 2004.

[5] U. Zakir, A. N. J. Leonce, and E. A. Edirisinghe, "Road Sign Segmentation Based on Colour Spaces A Comparative Study," Proc. International Conference on Computer Graphics and Imaging (CGIM), pp. $72-79,2010$

[6] S. Beucher and M. Bilodeau, "Road segmentation and obstacle detection by a fast watershed transformation," Proceedings of the Intelligent Vehicles Symposium, pp. 296- 301, 24-26 Oct. 1994.

[7] Y. Kang, K. Yamaguchi, T. Naito, and Y. Ninomiya, "Texture Segmentation of Road Environment Scene Using SfM Module and HLAC Features," IPSJ Transactions on Computer Vision and Applications, vol. 1, pp.220-230, 2009.

[8] G. Dong and X. Ming, "Road/traffic color image segmentation using RCE neural network," Intelligent robots and computer vision XVII : algorithms, techniques and active vision: proceedings of SPIE, vol. 3522, pp. 366-377, 1998

[9] S. Zheng, J. Liu, W. Shi, \& G. Zhu, "An automatic road segmentation algorithm using one-class SVM,” Proceedings of SPIE, vol. 6419, 2006. Retrieved from http://dx.doi.org/10.1117/12.713162.

[10] Prism Video Converter, http://www.nchsoftware.com/prism/index.html, Last accessed on January 2012.

[11] C. -C. Chang and C. -J. Lin, "LIBSVM: a library for support vector machines. ACM Transactions on Intelligent Systems and Technology," pp. 1-27, 2011. Software available at http://www.csie.ntu.edu.tw/ cjlin/libsvm.

\begin{tabular}{|c|c|c|c|c|c|c|c|c|c|c|c|c|}
\hline & lane & vege & sky & $\begin{array}{c}\text { road } \\
\text { surf }\end{array}$ & $\begin{array}{l}\text { White } \\
\text { board }\end{array}$ & $\begin{array}{l}\text { yellow } \\
\text { board }\end{array}$ & $\begin{array}{r}\text { board } \\
\text { blue }\end{array}$ & $\begin{array}{r}\text { board } \\
\text { green }\end{array}$ & $\begin{array}{r}\text { red } \\
\text { sign }\end{array}$ & $\begin{array}{r}\text { pole } \\
\text { gray }\end{array}$ & $\begin{array}{r}\text { board } \\
\text { black }\end{array}$ & pole_white \\
\hline lane & 76.923 & 23.077 & & & & & & & & & & \\
\hline vege & & 98.813 & & 0.29674 & 0.89021 & & & & & & & \\
\hline sky & & 5.5556 & 91.667 & & 2.7778 & & & & & & & \\
\hline road_surf & & 40.351 & & 59.649 & & & & & & & & \\
\hline white_board & 14.286 & 4.7619 & 9.5238 & & 71.429 & & & & & & & \\
\hline yellow_board & & 100 & & & & & & & & & & \\
\hline board_blue & & 100 & & & & & & & & & & \\
\hline board_green & & 75 & & & 25 & & & & & & & \\
\hline red_sign & & 62.5 & & & & & & & 37.5 & & & \\
\hline pole_gray & & 100 & & & & & & & & & & \\
\hline board_black & & 100 & & & & & & & & & & \\
\hline pole white & & 71.429 & & & 28.571 & & & & & & & \\
\hline
\end{tabular}

Figure 6: Segment classification accuracy using learning based framework 


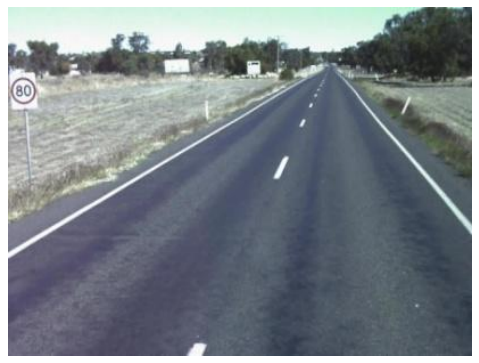

(a) Original image

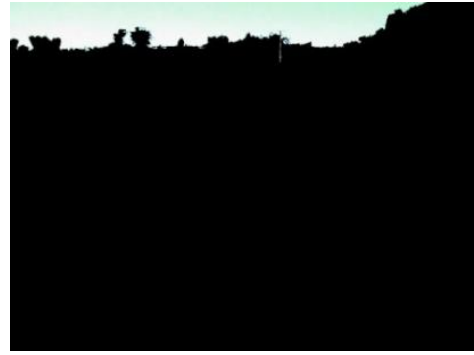

(c) Sky segment

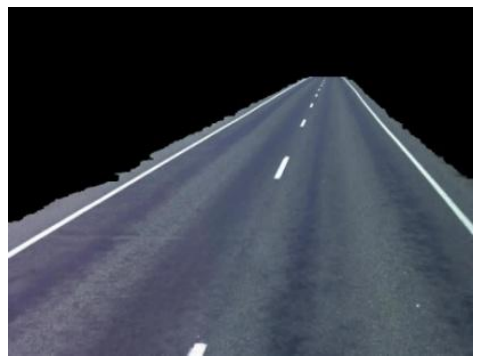

(b) Road segment

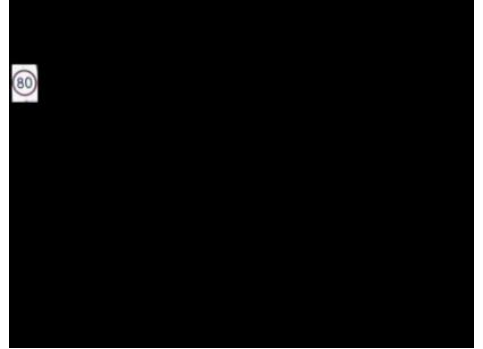

(d) Speed sign

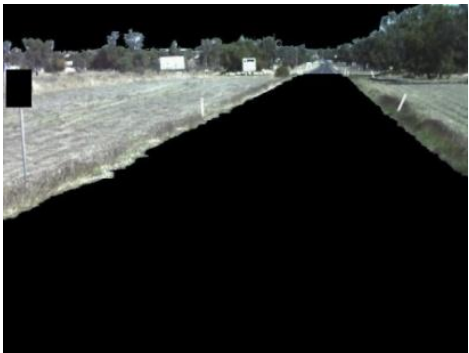

(e) Vegetation

Figure 7: Speed Sign, Road, Sky and Vegetation extraction.

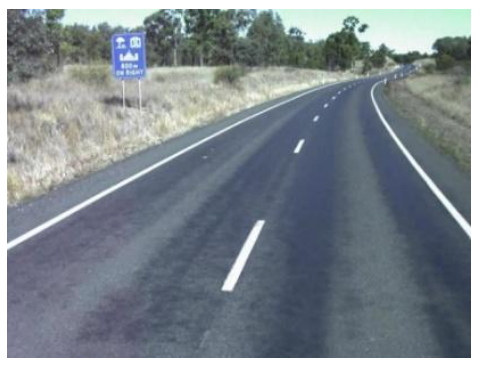

(a) Original image

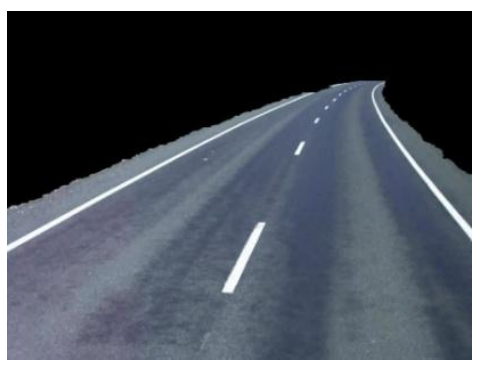

(b) Road segment

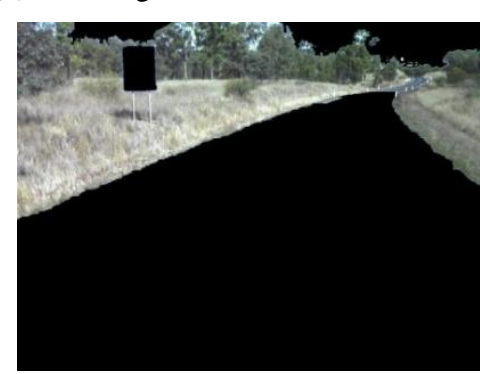

(e) Vegetation

(c) Sky segment

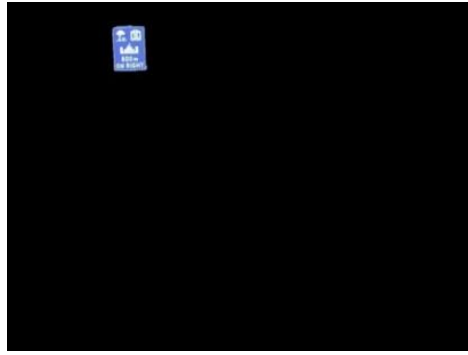

(d) Light blue sign

Figure 8: Light Blue Sign, Road, Sky and Vegetation extraction. 


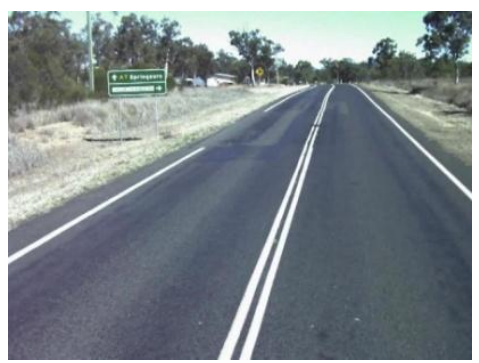

(a) Original image

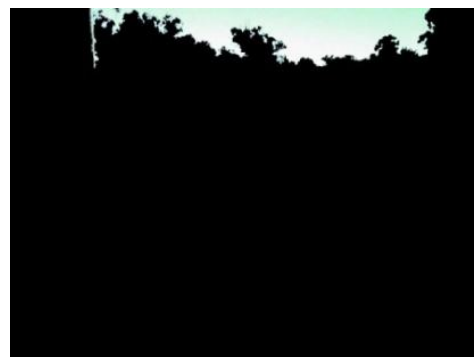

(c) Sky segment

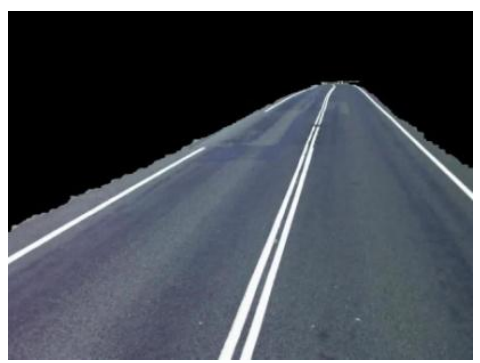

(b) Road segment

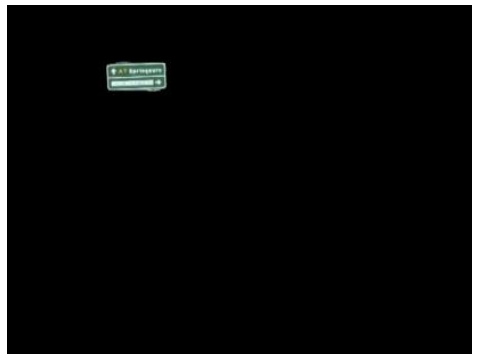

(d) Green sign

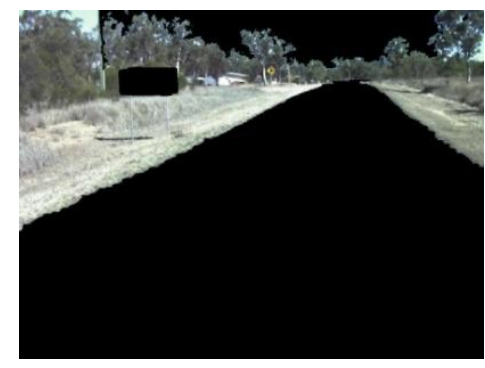

(e) Vegetation

Figure 9: Green Sign, Road, Sky and Vegetation extraction.

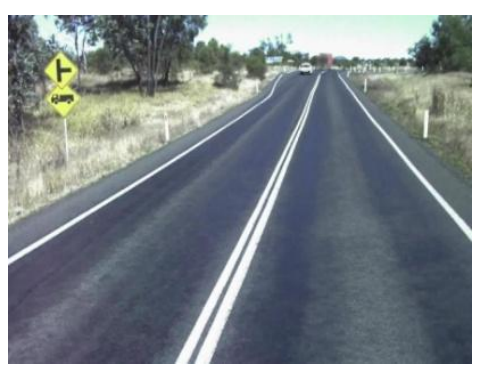

(a) Original image

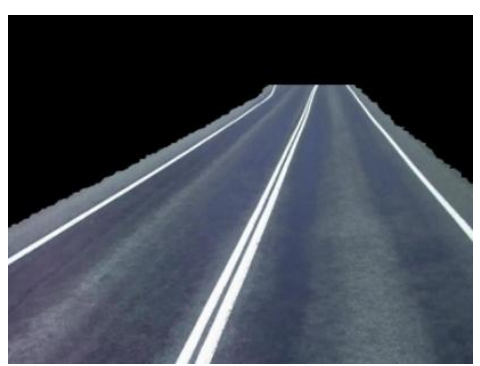

(b) Road segment

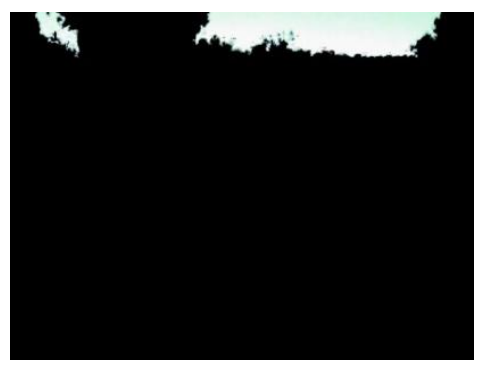

(c) Sky segment

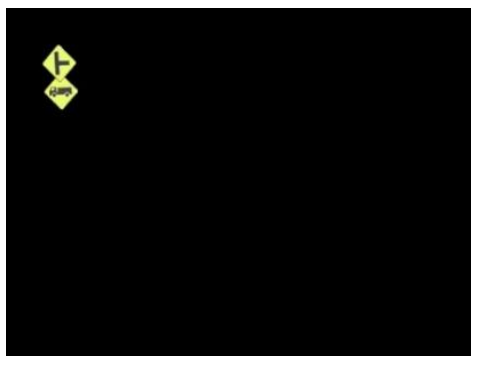

(d) Yellow sign

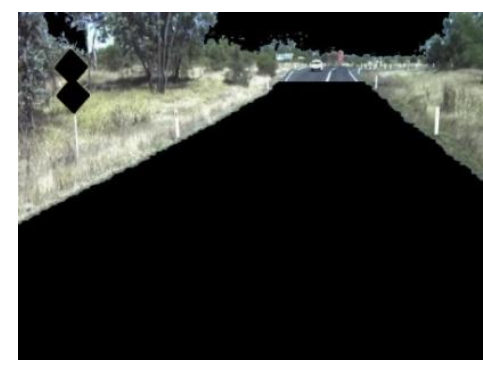

(e) Vegetation

Figure 10: Green Sign, Road, Sky and Vegetation extraction. 


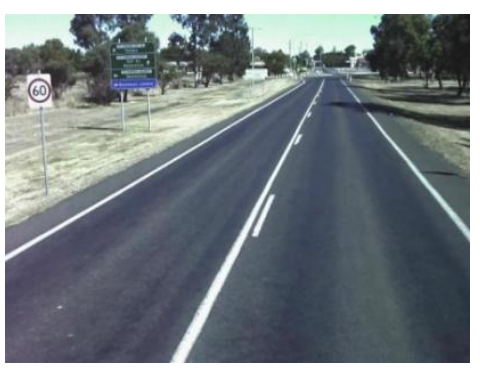

(a) Original image

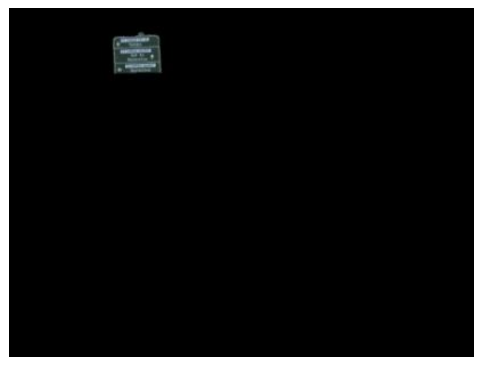

(d) Green sign

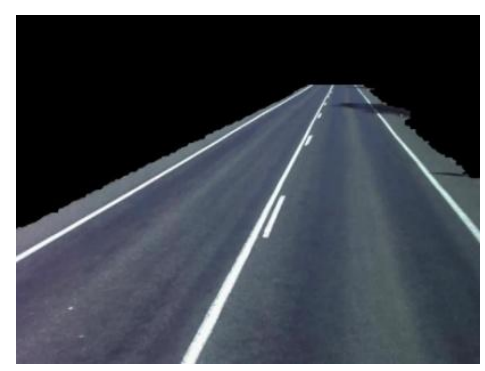

(b) Road segment

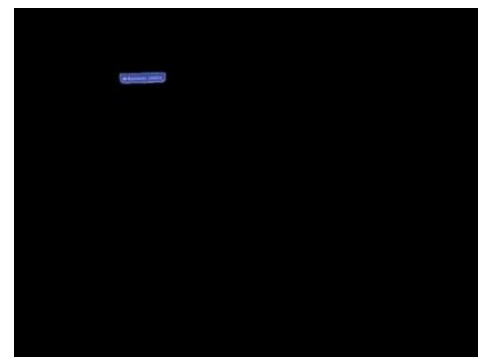

(e) Light blue sign

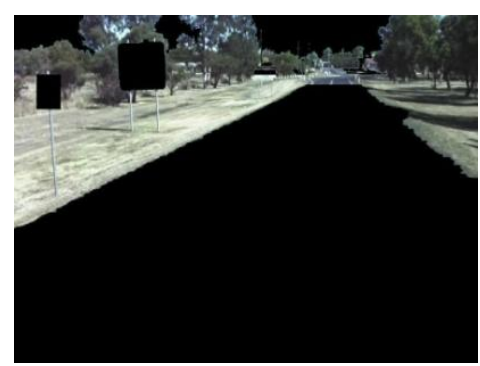

(g) Vegetation

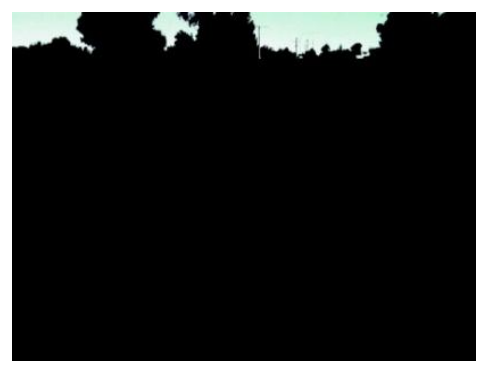

(c) Sky segment

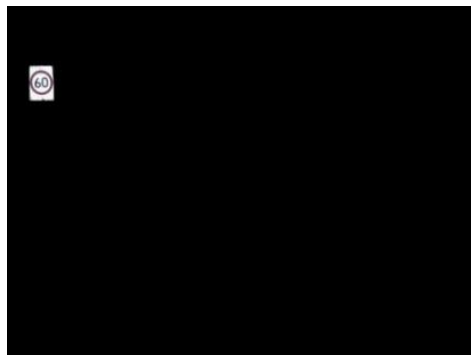

(f) Speed sign

Figure 11: Green Sign, Light Blue Sign, Speed sign, Road, Sky and Vegetation extraction 